\title{
FILTERS RESEARCH FOR FREE MOTION EXTRACTION IN SELF-TUNING AUTOMATIC CONTROL SYSTEMS
}

\author{
Khobin V.A. ${ }^{1}$, Levinskyi M.V. ${ }^{2}$ \\ ${ }^{1,2}$ Odessa National Academy of food technologies, Odessa \\ E-mail: ${ }^{2}$ MaxLevinskyi@gmail.com \\ ${ }^{2}$ ORCID: 0000-0002-6544-5110
}

Copyright (C 2014 by author and the journal "Automation technological and business - processes". This work is licensed under the Creative Commons Attribution International License (CC BY). http://creativecommons.org/licenses/by/4.0/

\begin{abstract}
Technological type control objects specific feature, which distinguish them among many mobile or electro technical types, is more low-frequency parametric disturbances spectral composition than spectral composition of coordinate disturbances. Most often parametric disturbances reveal themselves in changing control object transmission coefficient in the channel "controller control action - control variable". On a number of occasions transmission coefficient can change in a wide range - in $2 \ldots 10$ times more than initial value. Coordinate disturbances change control variables but don't change control object features. Besides spectral density of control variable change, under the impact of coordinate disturbances, has its peak in low-frequency range and characterize forced component of automatic control system motion. Parametric disturbances have influence on control variables and on control object properties, in particular on its transition coefficient. In this case, character of transient processes change, i.e. free motion component of the system. Spectral density of control variable changes, under the influence of transition coefficient change, has its peak, in general, in mid-frequency range. In the case, when using filters, even approximately, it is possible to separate out mid-frequency component from the overall process, which characterizes generally free motion of automatic control system, then it is possible to estimate current value of control object transmission coefficient using parameters change of this component. Such control variable components motions separation is used for designing selftuning ACS. In this case the informative parameter is dispersions difference of control object control variable and control variable of its model in the outputs of linear band pass filters. This dispersion difference is in proportion to current value of transition coefficient. In this article the results of simulation modeling computer experiments of self-tuning automatic control system with opened self-tuning loop are represented. The analysis of filtering efficiency, for three filter model options, are represented as well. The analysis was carried out with three different coordinate disturbances spectral compositions, with the same dynamics of control object and its model and with the different dynamics. Recommendations for filters structures alternatives selection are given. The possibility of optimal parametric tuning of filters is examined.
\end{abstract}

Key words

Free motion, filter, self-tuning, band pass filtering, automatic control system.

\section{Introduction}

A lot of factors influence technological process regulations variables when the process is considered as control object. These factors are represented as non-controlled coordinate and parametric disturbances. Coordinate disturbances change controlled variables without changing the properties of the control object. Parametric disturbances have influence on controlled variables and on the properties (parameters) of the control object as well. It is important to note that for control object of technological type the parametric disturbances spectral composition is much more low-frequency than spectral composition of coordinate disturbances [1,2]. This specific feature distinguishes control object of technological type from many mobile or electro-mechanical types.

Most often parametric disturbances reveal themselves in changing control object (CO) transmission coefficient in channel "controller control action - control variable". On a number of occasions transmission coefficient can change in a wide range in $2 \ldots 10$ times more than initial value. Transmission coefficient pattern change can be gradual, e.g. owing to deterioration of 
heat exchanger heat transfer due to accumulation of scale deposits. Or it can be rapid, like in the mills or presses when properties changing of feed stock occur.

In automatic control system (ACS) control variable $y(t)$ change in time relative to set point value $y^{s p}$ often is represented as sum of forced component $y_{f o r}(t)$, which is determined by specifics of external coordinate disturbances $f_{c}(t)$, measurement noises $f_{n}(t)$, which have influence on $\mathrm{CO}$, and free component $y_{f r}(t)$, which depends on parameters of $\mathrm{CO}$ and controller [3]:

$$
y(t)=y_{f o r}(t)+y_{f r}(t) \quad(1)
$$

Parametric disturbances $f_{p}(t)$, in particular, CO transmission coefficient $k_{o}(t)$ changes, usually, only scale forced component $y_{f o r}(t)$. But free component $y_{f r}(t)$ change pattern fundamentally changes: from protracted aperiodic transient processes during decrease of $k_{o}(t)$ and to oscillatory, including divergent, during increase of $k_{o}(t)$.

On the other hand control variable $y(t)$ time change can be represented as additive model of components with different spectral distribution [2, p. 33]:

$$
y(t)=\bar{y}(t)+y_{l}(t)+\tilde{y}(t)+y_{n}(t),
$$

where $\bar{y}(t)$ - constant or gradually changing component governed by $y^{s p}$ and $f_{c}(t)$. In particular case, when $y^{s p}=c o n s t$ and $f_{c}(t)$ $=$ const, then in ACS with offset absence $\bar{y}(t)=y^{s p}$;

$y_{l}(t)$ - low frequency component governed generally by influence of coordinate disturbances $f_{c}(t)$;

$\tilde{y}(t)$ - centered mid-range frequency component governed by free motion $y_{f r}(t)$ of ACS, as a matter of fact $\tilde{y}(t) \equiv y_{f r}(t)$;

$y_{n}(t)$ - high frequency component governed by, as a rule, measurement noises $f_{n}(t)$.

CO transmission coefficient $k_{o}(t)$ change reveal itself in $\tilde{y}(t)$ change. In the case, when using filters, even approximately, it is possible to separate out mid-frequency component $\tilde{y}(t)$ from the overall process $y(t)$, which characterizes generally free motion of ACS, then it is possible to estimate current value of CO transmission coefficient $k_{o}(t)$ using parameters change of $\tilde{y}(t)$. Such control variable components motions separation is used in [4] for designing self-tuning ACS. In that case $\tilde{y}(t)$ component was separated out by high frequency filters. But in that work there are no recommendations on choosing filters structures and parameters and also no optimization criteria is represented.

Problem statement

Let's define specifically the possibility of using of ACS free motion information for designing self-tuning ACS with CO varying transmission coefficient. For that, let's analyze block diagram extract of self-tuning ACS [4], which is shown in fig. 1. Self-tuning ACS includes ACS, which consists of CO with transfer function $W^{o}(s)$ and controller with transfer function $W^{c}(s)$, tunable model of CO with transfer function $W^{m}(s)$, two identical filters with transfer functions $W^{b f}(s)$ and two identical evaluators of probabilistic characteristics estimations Calc (here and further $s$ - differential operator). In this article controller of self-tuning circuit of $\mathrm{CO}$ model (parametric controller with transfer function $W^{p c}(s)$ ) is not discussed. The direction of action is shown by dashed line in the block diagram.

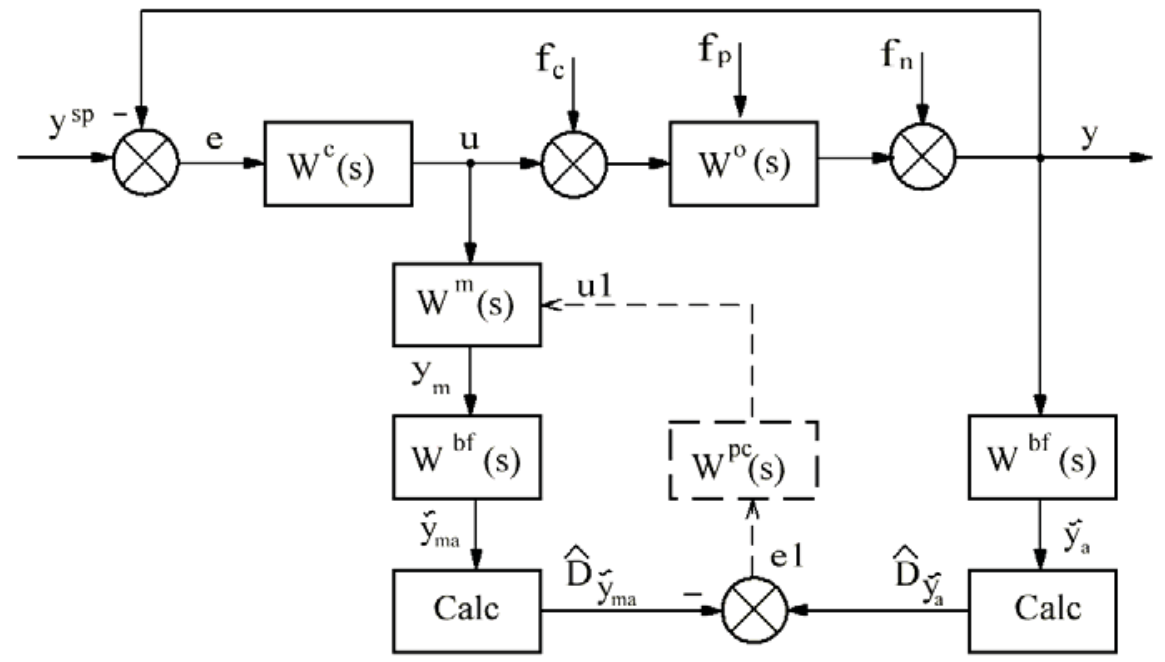

Fig. 1 - Self-tuning ACS block diagram extract

It is important to note that control variable $y(t)$ changes are developed by control action $u(t)$ of the stabilization circuit controller and by disturbances $f_{c}(t), f_{p}(t), f_{n}(t)$, whereas output variable of the tunable CO model $y_{m}(t)$ - only by influence of the control action $u(t)$. Since parametric disturbances $f_{p}(t)$ change more slowly than changes of $y(t)$ under the influence of 
$f_{c}(t)$ and $f_{n}(t)$, then it is possible to accept hypothesis about quasi-stationary character of CO parameters on the interval $T_{k s}$. Then the following motion equations, written in operator form, are true [5]:

$$
\begin{aligned}
& y(t)=W^{o}(s) \cdot u(t)+W^{o}(s) \cdot f_{c}(t)+f_{n}(t), \\
& y_{m}(t)=W^{m}(s) \cdot u(t),
\end{aligned}
$$

where controller control action $u(t)$ in the closed-loop ACS is defined as:

$$
u(t)=\frac{W^{c}(s)}{1+W^{o}(s) \cdot W^{c}(s)} \cdot\left[y^{s p}(t)-f_{n}(t)\right]-\frac{W^{o}(s) \cdot W^{c}(s)}{1+W^{o}(s) \cdot W^{c}(s)} \cdot f_{c}(t)
$$

The purpose of the filters is to eliminate (to filter out) all components from the signals $y(t)$ and $y_{m}(t)$ except centered midfrequency ones $\tilde{y}(t) \equiv y_{c}(t), \tilde{y}_{w}(t)$. In the output ports of the filters signals $\tilde{y}_{a}(t)$ and $\tilde{y}_{m a}(t)$ should be formed, which characterize the features of the $\tilde{y}(t)$ and the $\tilde{y}_{m}(t)$ accordingly:

$$
\begin{aligned}
& \tilde{y}_{a}(t)=W^{b f}(s) \cdot y(t)=W^{b f}(s) \cdot W^{o}(s) \cdot u(t)+W^{b f}(s) \cdot W^{o}(s) \cdot f_{c}(t)+W^{b f}(s) \cdot f_{n}(t) \\
& \tilde{y}_{m a}(t)=W^{m}(s) \cdot W^{b f}(s) \cdot u(t)
\end{aligned}
$$

In the time domain it is not rational to compare instantaneous signal values $\tilde{y}_{a}(t)$ and $\tilde{y}_{m a}(t)$ because: 1$)$ model always describes CO dynamic features only approximately; 2) real filters are not capable of ideal filtration of disturbances $f_{c}(t)$, $f_{n}(t)$ consequences; 3$)$ disturbances $f_{c}(t), f_{n}(t)$ points of applications to the $\mathrm{CO}$ and its model are different, therefore signals $\tilde{y}_{a}(t)$ and $\tilde{y}_{m a}(t)$ phases will not coincide. Possible solution to these problems is by using statistical averaging of $\tilde{y}_{a}(t)$ and $\tilde{y}_{m a}(t)$ variables and by comparing their stochastic characteristics, e.g. dispersions $D_{\tilde{y}_{a}}, D_{\tilde{y}_{m a}}$. Thematic example of this kind of approach is obtaining Wiener-Hopf equation from convolution equation.

Let's examine presentation of the dependences (5) - (7) in the frequency domain. In this way, control action $u(t)$ changes in time (5) is being changed, in accordance with [6], with its spectral density $S_{u}(\omega)$ :

$$
\begin{aligned}
S_{u}(\omega)= & \left|\frac{W^{c}(s)}{1+W^{o}(s) \cdot W^{c}(s)}\right|^{2} \cdot S_{y^{s p}}(\omega)-\left|\frac{W^{c}(s)}{1+W^{o}(s) \cdot W^{c}(s)}\right|^{2} \cdot S_{f n}(\omega)- \\
& -\left|\frac{W^{o}(s) \cdot W^{c}(s)}{1+W^{o}(s) \cdot W^{c}(s)}\right|^{2} \cdot S_{f c}(\omega)=\left|A^{y^{s p}-u}(j \omega)\right|^{2} \cdot S_{y^{s p}}(\omega)- \\
& -\left|A^{f n-u}(j \omega)\right|^{2} \cdot S_{f n}(\omega)-\left|A^{f c-u}(j \omega)\right|^{2} \cdot S_{f c}(\omega)
\end{aligned}
$$

where $S_{y^{s p}}(\omega)$ - spectral density of reference signal action $y^{s p}(t) ; S_{f c}(\omega)$ - spectral density of the coordinate disturbances $f_{c}(t)$ which is applied to the input of the $\mathrm{CO} ; S_{f n}(\omega)$ - spectral density of the noise $f_{n}(t)$, which is applied to the output of the CO; $A(j \omega)$ - frequency characteristics of the closed-loop ACS of according transfer channels. Dependences (6) and (7) assume the form:

$$
\begin{aligned}
S_{\tilde{y}_{a}}(\omega) & =\left|W^{o}(j \omega)\right|^{2} \cdot\left|W^{b f}(j \omega)\right|^{2} \cdot S_{u}(\omega)+ \\
& +\left|W^{o}(j \omega)\right|^{2} \cdot\left|W^{b f}(j \omega)\right|^{2} S_{f c}(\omega)+\left|W^{b f}(j \omega)\right|^{2} S_{f n}(\omega)= \\
& =A_{o}^{2}(\omega) \cdot A_{b f}^{2}(\omega) \cdot S_{u}(\omega)+A_{o}^{2}(\omega) \cdot A_{b f}^{2}(\omega) \cdot S_{f_{c}}(\omega)+ \\
& +A_{b f}^{2}(\omega) \cdot S_{f n}(\omega)=S_{\tilde{y}_{a}-u}(\omega)+S_{\tilde{y}_{a}-f c}(\omega)+S_{\tilde{y}_{a}-f n}(\omega)
\end{aligned}
$$




$$
S_{\tilde{y}_{m a}}(\omega)=\left|W^{m}(j \omega)\right|^{2} \cdot\left|W^{b f}(j \omega)\right|^{2} \cdot S_{u}(\omega)=A_{m}^{2}(\omega) \cdot A_{b f}^{2}(\omega) \cdot S_{u}(\omega),
$$

where $S_{\tilde{y}_{a}}(\omega)$ and $S_{\tilde{y}_{m a}}(\omega)$ - spectral densities in the outputs of the according filters; $W^{o}(j \omega)=k_{o} W^{o i}(j \omega)$, $W^{m}(j \omega)=k_{m} W^{m i}(j \omega)$ - CO transfer functions and its tunable model in the frequency domain; $W^{b f}(j \omega)$ - transfer function of the filters in the frequency domain; $S_{\tilde{y}_{a}-u}(\omega), S_{\tilde{y}_{a}-f c}(\omega), S_{\tilde{y}_{a}-f n}(\omega)$ - components of the spectral density $S_{\tilde{y}_{a}}(\omega)$, $A(\omega)$ - respective amplitude-frequency characteristics.

From (9) and (10) follows that spectral densities in the filters outputs $S_{\tilde{y}_{a}}$ and $S_{\tilde{y}_{m a}}$ are substantially different. That means they are not to be used in the task of tuning CO model transition coefficient $k_{m}(t)$ in accordance with variable CO transition coefficient $k_{o}(t)$ under the influence of the parametric disturbances $f_{p}(t)$. For solving this problem it is necessary to minimize the $S_{\tilde{y}_{a}-f c}(\omega)$ external coordinate disturbances $f_{c}(t)$ component contribution and $S_{\tilde{y}_{a}-f n}(\omega)$ component of the measurement noises $f_{n}(t)$ contribution into the $S_{\tilde{y} a}$ signal spectrum (9). This function is implemented in ACS by the bandpass filters. The structure and the parameters of the filters should be chosen so that spectral density in the filter output $S_{\tilde{y}_{a}}(9)$ is as much as possible close to spectral density $S_{\tilde{y}_{\text {ma }}}$ in the filter output (10).

By analogy with [4] let's show that values of dispersions $D_{\tilde{y}_{a}}, D_{\tilde{y}_{m a}}$ are dependable on CO transition coefficient $k_{o}$ and its model $k_{m}$, which, subject to (9)-(10), follows from these expressions:

$$
\left.\begin{array}{l}
D_{\tilde{y}_{a}}=\frac{1}{2 \pi} \int_{-\infty}^{\infty}\left[\left|W^{o}(j \omega)\right|^{2} \cdot\left|W^{b f}(j \omega)\right|^{2} \cdot S_{u}(\omega)+\right. \\
+\left|W^{o}(j \omega)\right|^{2} \cdot\left|W^{b f}(j \omega)\right|^{2} S_{f c}(\omega)+\left|W^{b f}(j \omega)\right|^{2} S_{f n}(\omega)
\end{array}\right] d \omega
$$

Transition coefficients $k_{o}, k_{m}$ are irrespective of frequency $\omega$. If it is possible, for the most part, to eliminate consequences of external disturbances $f_{c}(t)$ и $f_{n}(t)$ actions on the control variable $y(t)$ using filtration, to make them insignificant, then in (11) components $\left|W^{o}(j \omega)\right|^{2} \cdot\left|W^{b f}(j \omega)\right|^{2} S_{f c}(\omega) \rightarrow 0,\left|W^{b f}(j \omega)\right|^{2} S_{f n}(\omega) \rightarrow 0$. Consequently, the expressions (11) and (12) assume the form:

$$
\begin{aligned}
& D_{\tilde{y}_{a}} \approx k_{o}^{2} \frac{1}{2 \pi} \int_{-\infty}^{\infty}\left|W^{o i}(j \omega)\right|^{2} \cdot\left|W^{b f}(j \omega)\right|^{2} \cdot S_{u}(\omega) d \omega \\
& D_{\tilde{y}_{m a}}=k_{m}^{2} \frac{1}{2 \pi} \int_{-\infty}^{\infty}\left|W^{m i}(j \omega)\right|^{2} \cdot\left|W^{b f}(j \omega)\right|^{2} \cdot S_{u}(\omega) d \omega
\end{aligned}
$$

From (13) and (14) follows that if CO model $W^{m i}(j \omega)$ adequately defines dynamics of the real CO $W^{o i}(j \omega)$, i.e. $W^{o i}(j \omega) \approx W^{m i}(j \omega)$, then with rough equality of signals dispersions in the filters outputs $D_{\tilde{y}_{a}}(t) \approx D_{\tilde{y}_{m a}}(t)$, transition coefficients of the $\mathrm{CO}$ and its model will be roughly equal as well $k_{o}^{2} \approx k_{m}^{2}$. Exactly this fact is being used when designing self-tuning ACS in [4].

In accordance with accepted hypothesis about $\mathrm{CO}$ parameters quasi-stationary in the $T_{k s}$ interval, changes of transition coefficient $k_{o}(t)$ happen relatively slowly. This makes it necessary and gives opportunity to calculate dispersions estimations (13), (14) in sliding time interval $\tau_{a v} \leq T_{k s}$. In the real time these estimations are obtained, usually, by applying exponentially weighted average or evenly weighted average to stochastic processes, in this case $\tilde{y}_{a}^{2}(t)$ and $\tilde{y}_{m a}^{2}(t)$, "passing" them through according dynamic links with transfer functions $W^{e v w}(s)=\frac{\left(1-\exp \left(-\tau_{a v} s\right)\right)}{\tau_{a v} s}$ or $W^{\exp w}(s)=\frac{1}{0,5 \tau_{a v} s+1}$ [7]: 


$$
\begin{aligned}
& \hat{D}_{\tilde{y}_{a}}\left(t, \tau_{a v}\right)=\frac{\left(1-\exp \left(-\tau_{a v} s\right)\right)}{\tau_{a v} s} \cdot \tilde{y}_{a}^{2}(t), \hat{D}_{\tilde{y}_{m a}}\left(t, \tau_{a v}\right)=\frac{\left(1-\exp \left(-\tau_{a v} s\right)\right)}{\tau_{a v} s} \cdot \tilde{y}_{m a}^{2}(t), \\
& \hat{D}_{\tilde{y}_{a}}\left(t, \tau_{a v}\right)=\frac{1}{0,5 \tau_{a v} s+1} \cdot \tilde{y}_{a}^{2}(t), \quad \hat{D}_{\tilde{y}_{m a}}\left(t, \tau_{a v}\right)=\frac{1}{0,5 \tau_{a v} s+1} \cdot \tilde{y}_{m a}^{2}(t)
\end{aligned}
$$

In this paper the evaluators Calk in the block diagram (fig. 1) calculate the estimations using dependences (16), because they are easier to implement in a program.

It is important to note that estimations difference $e 1\left(t, \tau_{a v}\right)=\hat{D}_{\tilde{y}_{a}}\left(t, \tau_{a v}\right)-\hat{D}_{\tilde{y}_{m a}}\left(t, \tau_{a v}\right)$, same as dispersions difference $D_{\tilde{y}_{a}}-D_{\tilde{y}_{m a}}$, is proportional to difference $k_{o}^{2}-k_{m}^{2}$. Changes in dispersion estimation $\hat{D}_{\tilde{y}_{a}}\left(t, \tau_{a v}\right)$ indicates the beginning of $\mathrm{CO}$ transition coefficient $k_{o}(t)$ changes. Hence it is obvious that variable $e 1\left(t, \tau_{a v}\right)$ can be selected as an informative variable of $\mathrm{CO}$ model transition coefficient $k_{m}(t)$ self-tuning circuit in relation with varying CO transition coefficient $k_{o}(t)$.

From the abovementioned follows that accuracy of the self-tuning procedure in self-tuning ACS depends on the filters operation quality, their capability of division forced component of the ACS motion from the free component. Objective quality indicator in this case can be statistical correlation coefficient, which provide information about level of closeness between variables $\tilde{y}_{a}(t)$ and $\tilde{y}_{m a}(t)$ in the filters outputs.

The goal of this article - to define the approach of filters structure and parameters selection for self-tuning ACS, simplified for the present, i.e. with disconnected self-tuning circuit, and also to compare filtration efficiency, using statistical correlation coefficient, of the variables in the filter outputs in the time of different mismatches between $\mathrm{CO}$ and its model and during different spectral densities of coordinate disturbances.

Conditions for carrying out comparative research

Computer modeling was chosen as an instrument for the research, because analytical approach towards solving analysis and synthesis problems of non-stationary ACS, with varying transition coefficients objects, have approximate character and were obtained for separate particular cases [5, p. 397].

- Options of CO dynamics descriptions - transfer functions of the "virtual" CO:

$$
W^{o}(s)=\frac{k_{o}}{\left(T 1_{o} s+1\right)^{4}\left(T 2_{o} s+1\right)^{2}} \exp \left(-\tau_{o} s\right)=\frac{1}{(0,05 s+1)^{4}(1,27 s+1)^{2}} \exp (-0,1 s)
$$

being approximated by models of different degrees:

$$
\begin{gathered}
W^{o}(s)=\frac{k_{o}}{\left(T_{o} s+1\right)} \exp \left(-\tau_{o} s\right)=\frac{1}{2 s+1} \exp (-1 s) \\
W^{o}(s)=\frac{k_{o}}{\left(T_{o} s+1\right)^{2}} \exp \left(-\tau_{o} s\right)=\frac{1}{(1,27 s+1)^{2}} \exp (-0,3 s), \\
W^{o}(s)=\frac{k_{o}}{\left(T 1_{o} s+1\right)^{2}\left(T 2_{o} s+1\right)} \exp \left(-\tau_{o} s\right)=\frac{1}{(0,61 s+1)^{2}(1,6 s+1)} \exp (-0,1 s),
\end{gathered}
$$

where: $k_{o}, \tau_{o}, T_{o}, T 1_{o}, T 2_{o}$ - values of the transition coefficient, time delay and time constants, which has been evaluated using recommendations [8]. Step responses of the "virtual" control object are represented in the fig. 2a.

- Controller

$$
W^{c}(s)=k_{r} \cdot\left(1+1 / T_{i z} s\right),
$$

where: $k_{r}=2,3 ; T_{i z}=2,7$ - values of the transition coefficient and integral action time, which corresponds to optimal value of the integral squared criterion of the control error for the CO option (18). Step responses of the ACS with the controller (21) and different options of the "virtual" $\mathrm{CO}$ are represented in the fig. $2 \mathrm{~b}$. 


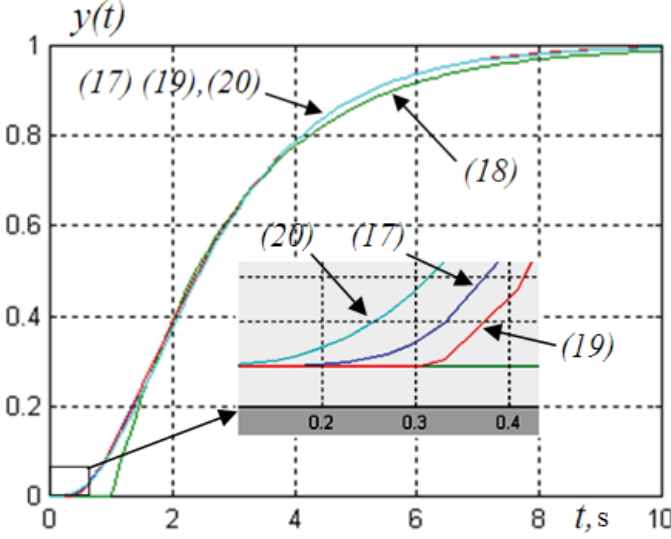

a)

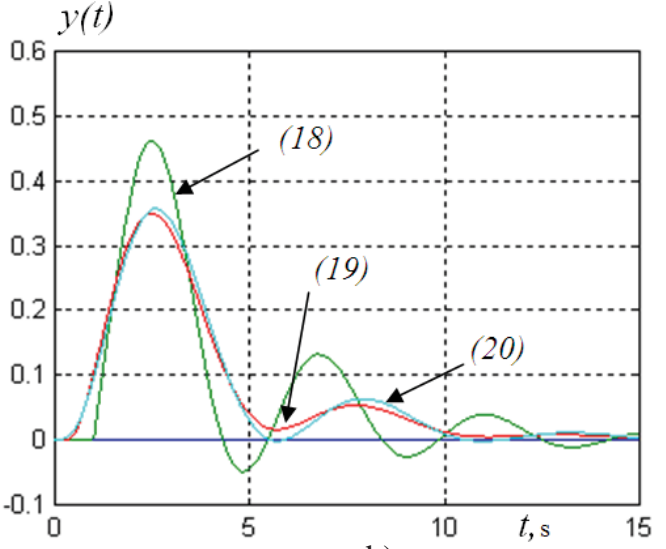

b)

Fig. 2 - Step responses:

a) different options of the $\mathrm{CO}$;

b) ACS with the different options of the "virtual" $\mathrm{CO}$

- models of coordinate disturbances in the $\left\langle f_{c^{-}} y »\right.$ channel: $f_{c}(t)=m_{f_{c}}+\tilde{f}_{c}(t)$ :

$$
\begin{aligned}
& m_{f c}=1, S_{\tilde{f c}_{c}}(\omega)=1 /\left(1+(\omega / 0,25)^{4}\right), \\
& m_{f c}=1, S_{\tilde{f c}}(\omega)=1 /\left(1+(\omega / 0,5)^{4}\right), \\
& m_{f c}=1, S_{\tilde{f} c}(\omega)=1 /\left(1+(\omega / 0,75)^{4}\right),
\end{aligned}
$$

where: $m_{f c}$ - constant component of the $f_{c}(t), S_{\tilde{f} c}(\omega)$ - spectral density of the stochastic component $\tilde{f}_{c}(t)$. Stochastic components $\tilde{f}_{c}(t)$ were formed using filter with the transfer function $W^{f f}(s)=\omega_{g f}^{2} /\left(s^{2}+\sqrt{2} \cdot \omega_{g f} s+\omega_{g f}^{2}\right)$ from the pseudo white noise signal with the cut-off frequencies: $\omega_{g f} \in 0,25 ; 0,5 ; 0,75 \mathrm{rad} / \mathrm{s}$. Stochastic processes realizations options $\tilde{f}_{c}(t)$ and their statistical characteristics are represented in fig. 3.

Stochastic processes realizations Probability densities estimations

(22)

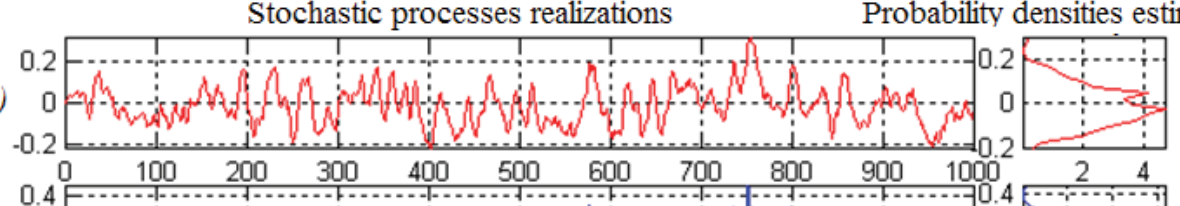

(23) 0

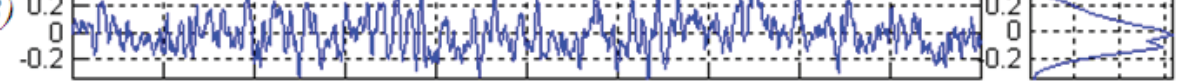

\section{(24)}

\begin{tabular}{|c|c|c|c|}
\hline \multirow{2}{*}{ Numerical estimations in the whole modeling interval } & \multicolumn{3}{|c|}{ Process number } \\
\hline & 1 & 2 & 3 \\
\hline Estimation of the average of distribution & 0 & 0 & 0 \\
\hline Estimation of the mean square deviation & 0.09 & 0.13 & 0.16 \\
\hline Estimation of the mean square period / frequency & $29.0 / 0.22$ & $11.8 / 0.53$ & $/ 0.77$ \\
\hline
\end{tabular}
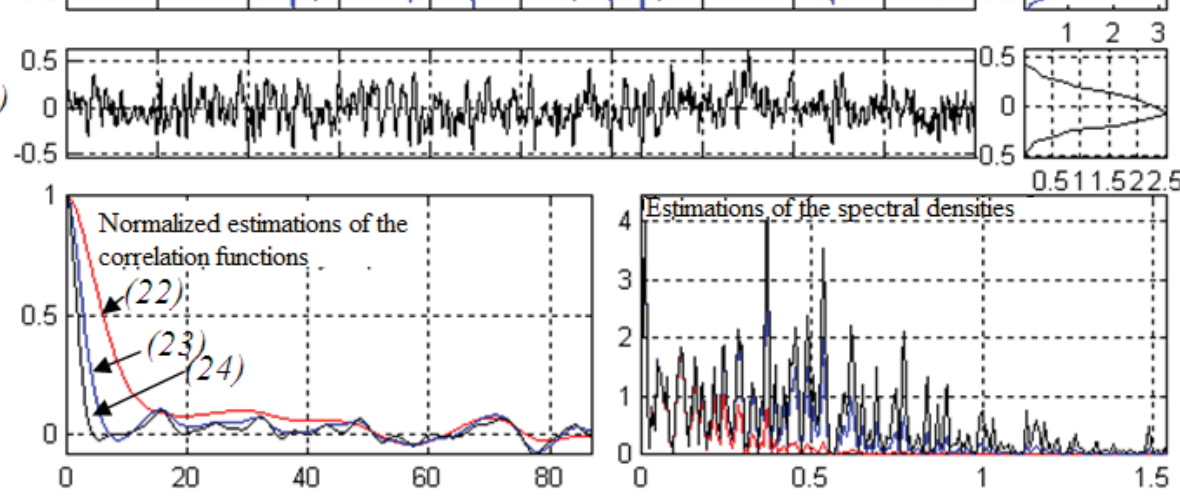

Fig. 3 - Realizations of the stochastic processes (22), (23), (24) - options of the coordinate disturbances $\tilde{f}_{c}(t)$ and estimations of their stochastic functions 
For checking of the fact that the requirement was met, which was pointed out in the introduction to the article, (see relation (2)), about low-frequency character of the coordinate disturbances $\tilde{f}_{c}(t)$ in comparison to free motion

of the ACS, which can be judged by its amplitude-frequency characteristic (AFC), it is enough to examine fig. 4. Here it is necessary to note, that disturbances modes (22), (23) can be taken as low-frequency in comparison to AFC of the ACS, and the model (24) was ad hoc was chosen with the violation of this condition.

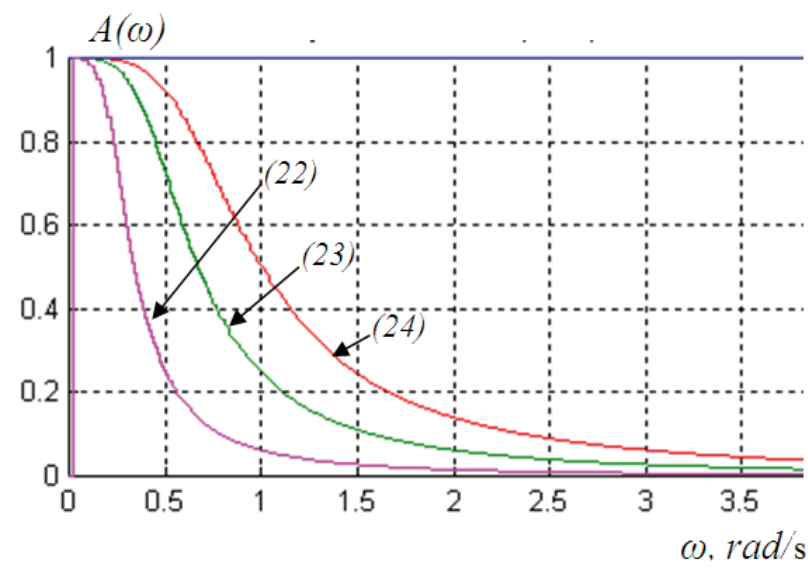

a)

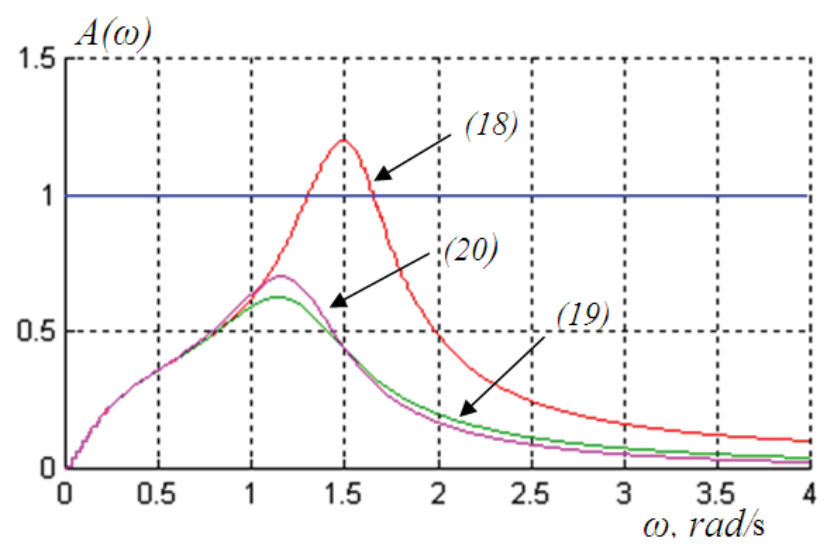

b)

Fig. 4 - AFC: a) of the filters (22), (23), (24) which form $\tilde{f}_{c}(t)$

b) closed-loop ACS through the channel $« f_{c}-y$ » with different options of the "virtual" control object

- Tunable control object model in the self-tuning circuit (the same for all options of the "virtual" CO):

$$
W^{m}(s)=\frac{k_{m}}{\left(T_{m} s+1\right)} \exp \left(-\tau_{m} s\right)
$$

Two approaches are used: a) idealized conditions, when parameters (25) coincide with the parameters (18); b) more realistic conditions, when $\tau_{m}=0,7 \cdot \tau_{o}=0,7 \mathrm{~s}$ or $\tau_{m}=1,3 \cdot \tau_{o}=1,3 \mathrm{~s}$. In the real conditions time delay in the CO can change, but when modeling it is more convenient to change the time delay in the model of the CO, which can be interpreted as not accurate reflection of the CO properties in the model. Dynamic properties of the ACS remain constant.

- Transfer functions of the linear band pass filters options - Butterworth filters, which have AFC with horizontal area in the pass band [9]:

$$
\begin{aligned}
& \text { a) } W_{1}^{b f}(s)=\frac{s}{s+\omega_{H}} \cdot \frac{1}{s+\omega_{L}} \text {; } \\
& \text { b) } W_{2}^{b f}(s)=\frac{s^{2}}{s^{2}+1,414 \cdot \omega_{H} s+\omega_{H}^{2}} \cdot \frac{\omega_{L}^{2}}{s^{2}+1,414 \cdot \omega_{L} s+\omega_{L}^{2}} \text {; } \\
& W_{3}^{b f}(s)=\frac{s^{2}}{s^{2}+1,848 \cdot \omega_{H} \cdot s+\omega_{H}^{2}} \cdot \frac{s^{2}}{s^{2}+0,765 \cdot \omega_{H} \cdot s+\omega_{H}^{2}} \cdot \\
& \cdot \frac{\omega_{L}^{2}}{s^{2}+1,848 \cdot \omega_{L} \cdot s+\omega_{L}^{2}} \cdot \frac{\omega_{L}^{2}}{s^{2}+0,765 \cdot \omega_{L} \cdot s+\omega_{L}^{2}}
\end{aligned}
$$

where $\omega_{H}, \omega_{L}$ - cut-off frequencies of the filters in the low-frequency and high-frequency ranges. These frequencies will be taken as input parameters of the filter tuning optimization procedure.

$$
\omega_{H}^{*}, \omega_{L}^{*}=\arg \max r_{\tilde{y} \tilde{y}_{m}}\left(W_{i}^{b f}(s), \omega_{H}, \omega_{L}\right)
$$

In the fig. 5 step responses of the filters (26)-(28) are shown, as well as their AFC. 

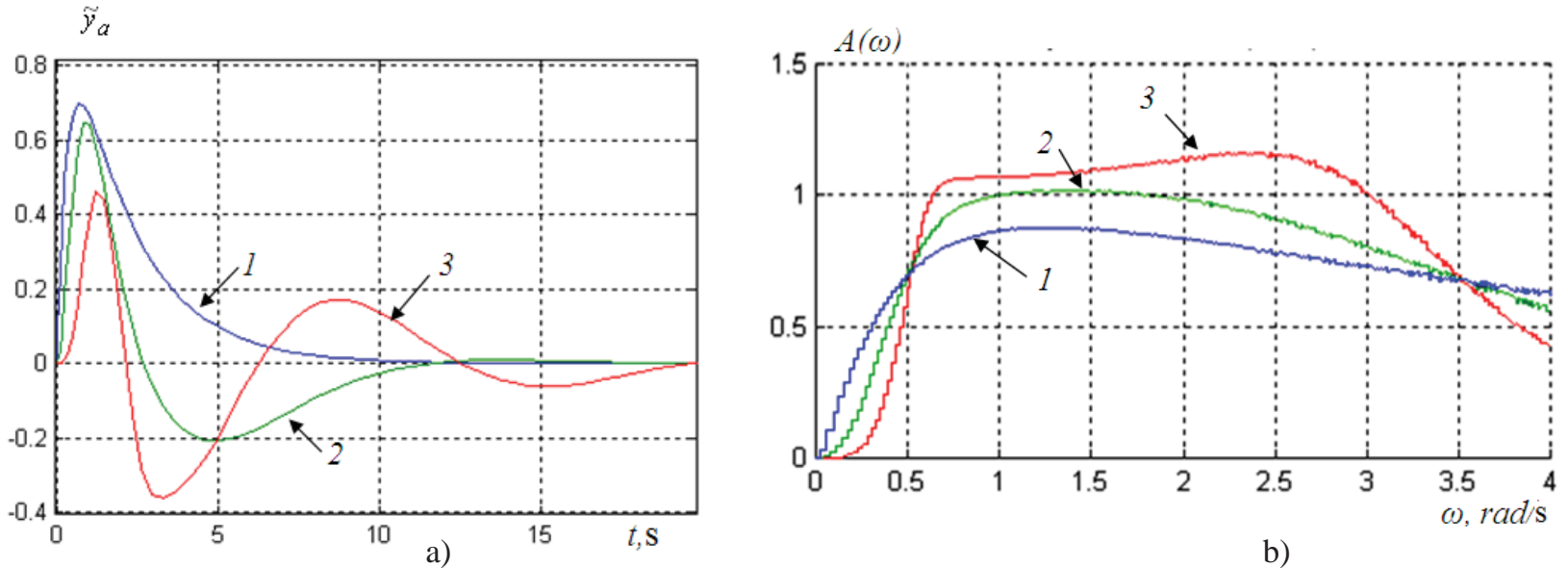

b)

Fig. 5 - a) step responses and b) AFC of the band pass filters with the cut-off frequencies $\omega_{H}=0.5, \omega_{L}=3: 1-$ filter (26), $2-(27), 3-(28)$

- Criterion for the optimization procedure of the filters tuning - the proximity level of the variables in the filters outputs $\tilde{y}_{a}(t)$ and $\tilde{y}_{m a}(t)$ which was evaluated using statistical correlation coefficient [5, p. 410]:

$$
\hat{r}_{\tilde{y}_{a} \tilde{y}_{m a}}=\frac{\hat{R}_{\tilde{y}_{a} \tilde{y}_{m a}}\left(\tau_{k}=0\right)}{\hat{\sigma}_{\tilde{y}_{a}} \cdot \hat{\sigma}_{\tilde{y}_{m a}}}=\frac{\frac{1}{T_{\bmod }} \int_{t 1}^{t 1+T \bmod } \tilde{y}_{a}(t) \cdot \tilde{y}_{m a}(t) d t}{\sqrt{\frac{1}{T_{\bmod }}} \cdot \int_{t 1}^{t 1+T \bmod } \tilde{y}_{a}^{2}(t) d t \cdot \sqrt{\frac{1}{T_{\bmod }}} \cdot \int_{t 1}^{t 1+T \bmod } \tilde{y}_{m a}^{2}(t) d t}=\frac{\int_{t 1}^{t 1+T \bmod } \tilde{y}_{a}(t) \cdot \tilde{y}_{m a}(t) d t}{\sqrt{\int_{t 1}^{t 1+T \bmod } \tilde{y}_{a}^{2}(t) d t} \cdot \sqrt{\int_{t 1}^{t 1+T \bmod } \tilde{y}_{m a}^{2}(t) d t}}
$$

where in the interval $\mathrm{T}_{\text {mod }}$ were evaluated estimations: $\hat{R}_{\tilde{y}_{a}} \tilde{y}_{m a}\left(\tau_{k}=0\right)$ - correlation moment with no phase shift between the processes; $\hat{\sigma}_{\tilde{y}_{a}}, \hat{\sigma}_{\tilde{y}_{m a}}$ - mean square deviations of the variables $\tilde{y}_{a}(t)$ and $\tilde{y}_{m a}(t) ; t 1$ - beginning of the calculations point. It is obvious that the better the filtering of the $\mathrm{CO}$ control variable $y(t)$ and its model $y_{m}(t)$ from the consequences of the disturbances and the noises impacts, the higher the value of the statistical correlation coefficient $\hat{r}_{\tilde{y}_{a}} \tilde{y}_{m a}$ will be.

\section{Planning of the computer experiments}

The goal of carrying out experiments is to research the effectiveness of ACS free motion extraction using the band pass linear filtering. The evaluation of the effectiveness was carried out using (30). Computer experiments intend purposive simulation modeling of the block diagram in fig. 1 in the Simulink environment of the MatLab programs package. ACS options with the "virtual" CO models (18), (19), (20) which were examined, under the influence of coordinate disturbances $f_{c}(t)$ with the models options (22), (23), (24) for idealized conditions with $\tau_{m}=\tau_{o}=1 \mathrm{~s}$ and with mismatch of time delays $\tau_{m}=0,7$ or $\tau_{m}=1.3$. Simulation model is shown in fig 6.

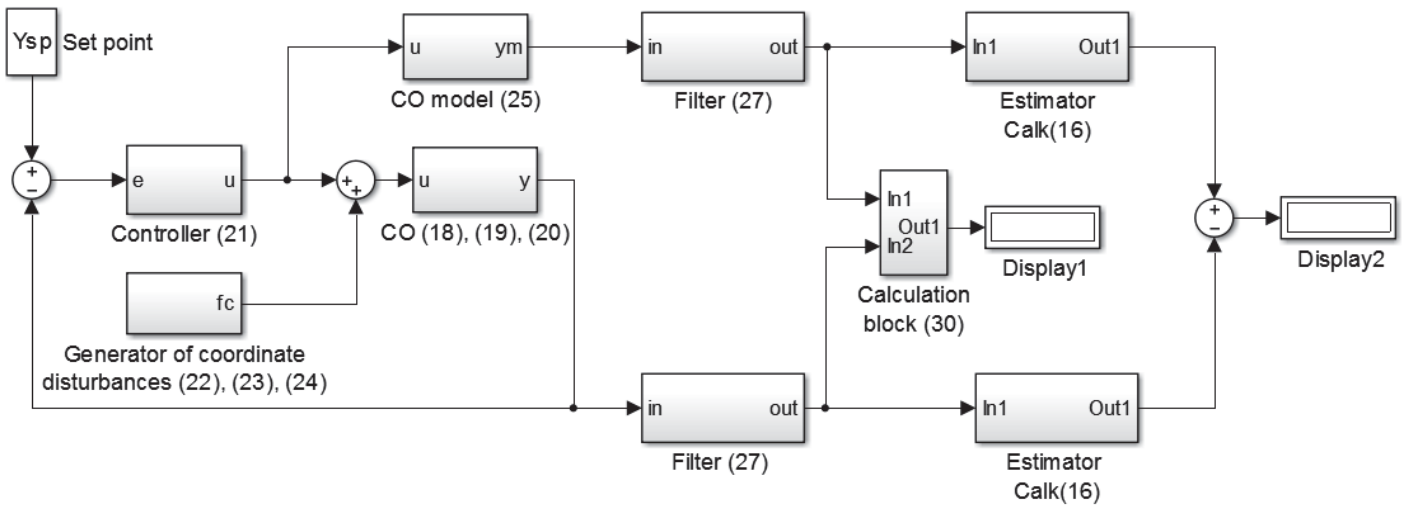

Fig. 6 - Simulation model for evaluation of effectiveness of the variables $\tilde{y}_{a}(t)$ and $\tilde{y}_{m a}(t)$ filtering (in the brackets there are numbers of mathematical relations which are implemented by corresponding blocks) 
Rationale of the linear band pass filters parameters and structure expediency, filtration efficiency comparative research

Selection of the appropriate filter structure was carried out from the alternative options (26), (27), (28) for frequency range $\omega_{H}=0,5 \div \omega_{L}=3,0$, in which the free motion of the examined ACS is located in general. In fig. 7 the examples of ACS variables realization in time domain are shown, from which the physical meaning of $y(t), y_{m}(t)$ filtration from the consequences of coordinate disturbances $f_{c}(t)$ impact is clearly seen. The filters have to "bring together" output variables $\tilde{y}_{a}(t)$ and $\tilde{y}_{m a}(t)$ in comparison to the state of the output variables $y(t)$ и $y_{m}(t)$. The values of the correlation coefficient $\hat{r}_{\tilde{y}_{a}} \tilde{y}_{m a}$, which provides numeric characteristic of filtration efficiency, i.e. the level of $\tilde{y}_{m a}(t)$ proximity to $\tilde{y}_{a}(t)$, are shown later in the table 1.
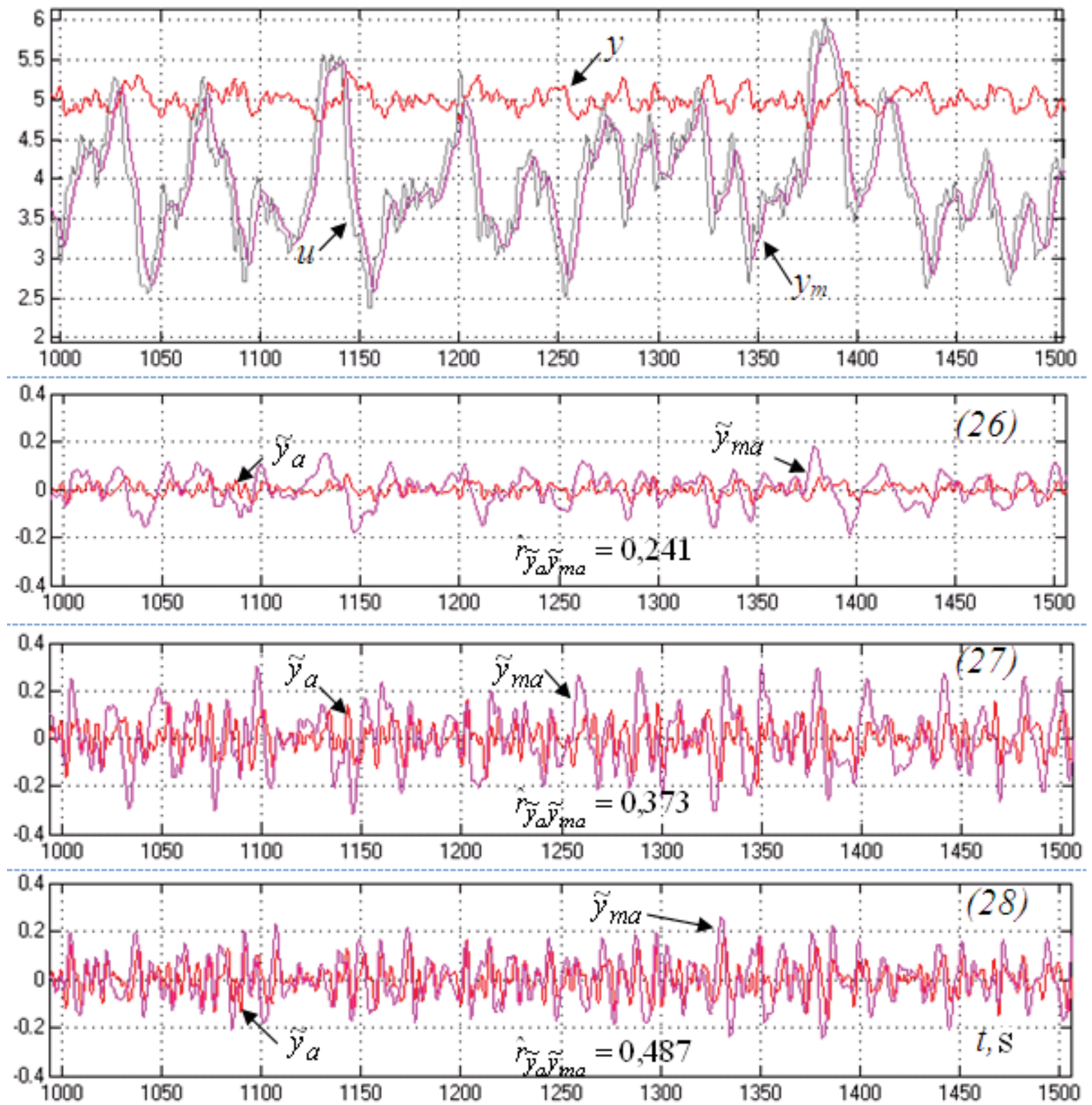

Fig. 7 - The examples of the ACS variables realizations in the time domain for the filters options (26), (27), (28), CO (18), $f_{c}(t)(22)$

From fig. 7 follows that the second order filter (26) has worst suppresions qualities of the $f_{c}(t)$ consequences, because in the $\tilde{y}_{m a}(t)$ variable change one can see low-frequency components and that's why it can be excluded from the consideration at once.

Filter (28) is better than the others in ragard to suppression of the $f_{c}(t)$ consequences and the spectral density of the variables 
$\tilde{y}_{a}(t)$ и $\tilde{y}_{\text {та }}(t)$ is very close. But technical implementation of the eighth order filter (28) is much more complex than the fourth order filter (27) but quality and quantity filtering efficiency indicators for these two structures are close. As an additional verification for linear band pass filter (27) in the frequency domain can be fig. 8, in which AFC of ACS with selftuning using this filter through channels $f_{c}-y, f_{c^{-}} y_{m}$ and $f_{c}-\tilde{y}_{a}, f_{c}-\tilde{y}_{m a}$.
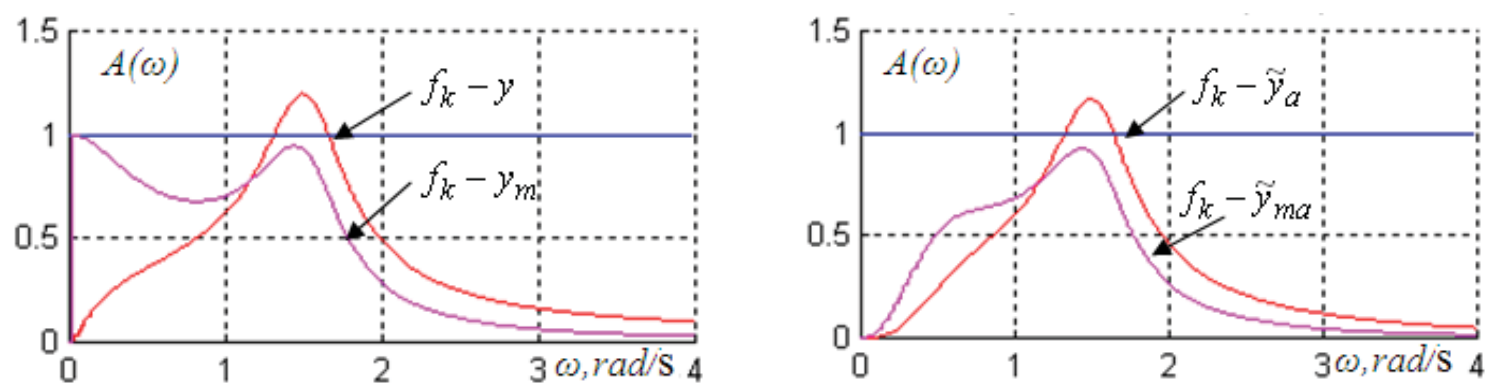

Fig. 8 - AFC of the system with the filter (27) trough channels

$f_{c}-y, f_{c^{-}} y_{m}$ и $f_{c}-\tilde{y}_{a}, f_{c}-\tilde{y}_{m a}$, «virtual» $\mathrm{CO}(18)$

As follows from fig. 8, linear band pass filter (27) provides sufficient for practical applications AFC through the channel $f_{c}-\tilde{y}_{m a}$ level of proximity to the AFC through the channel $f_{k}-\tilde{y}_{a}$. Because of that hereinafter it is rational to use filters of not less than the fourth order.

Parameters tuning of linear band pass filter (27) assumes search using (29) of optimal cut-off frequencies in lowfrequency and high-frequency ranges $\omega_{H}^{*}, \omega_{L}^{*}$. The initial values of these frequencies can be selected using following recommendations.

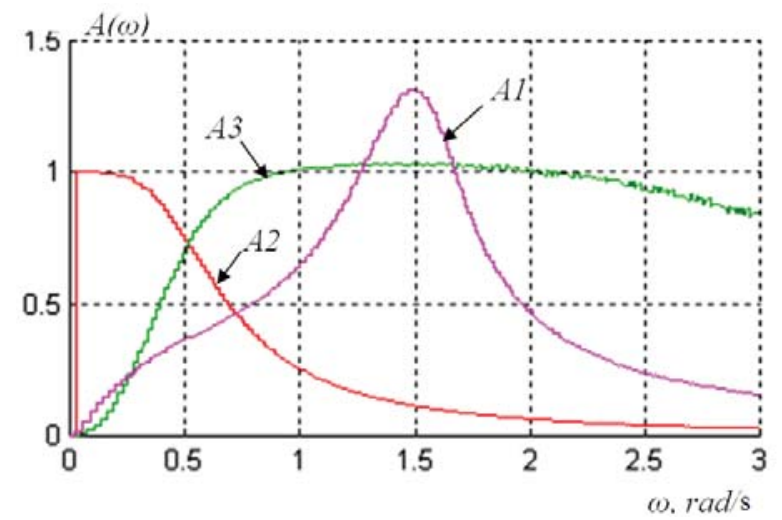

Fig. 9 - AFC: A1 - closed-loop ACS with CO (18) through the channel «f $f_{c}^{-} y »$;

$\mathrm{A} 2$ - of filter (23), forming $f_{c}$; $\mathrm{A} 3$ - of band pass filter (27)

As follows from fig. 9, cut-off frequency of low-frequency range can be set equal to frequency, on which spectral density of disturbance $\tilde{f}_{k}(t)$ getting less by one third of its original value, i.e. $\omega_{H} \approx \omega_{A_{2}}(\omega)=0,7$; cut-off frequency of the highfrequency range can be set on the frequency on which AFC of the closed-loop ACS through the disturbances channel gets less until value of 0,25 , i.e. $\omega_{L} \approx \omega_{A_{1}(\omega)=0,25}$. In the pass band linear band pass filter must ensure transition coefficient, which is close to one.

Before optimization procedure research of the dependence $\hat{r}_{\tilde{y}_{a}} \tilde{y}_{m a}=f\left(\omega_{H}, \omega_{L}\right)$ was carried out. In fig. 10 examples of this dependence for two ACS with self-tuning options with linear band pass filter (27) are shown. Changes of this function for other cases in table 1 have similar character. 


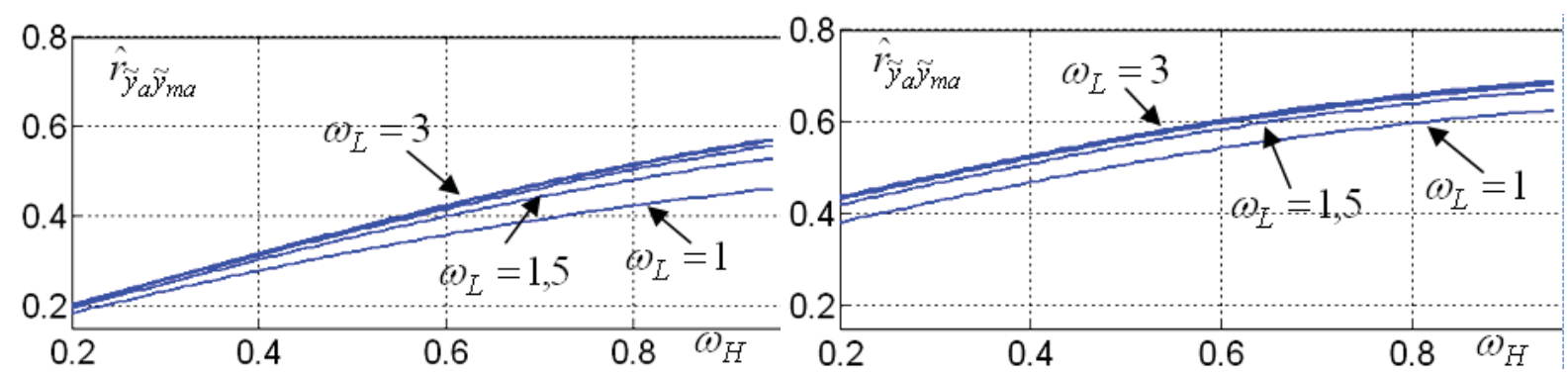

Fig. 10 - Plots $\hat{r}_{\tilde{y}_{a} \tilde{y}_{m a}}=f\left(\omega_{H}, \omega_{L}\right)$ for ACS with self-tuning with linear filter (27) with $\tau_{m}=1 \mathrm{~s}$ :

a) $\mathrm{CO}(18), f_{c}(22)$; b) $\mathrm{CO}(20), f_{c}(24)$;

Considering the outlook of the plots, one can make conclusion, that carrying out unconstrained parametric optimization of the function $\hat{r}_{\tilde{y}_{a}} \tilde{y}_{m a}=f\left(\omega_{H}, \omega_{L}\right)$ is not considered as possible, because its extreme is situated in the limit of examined parameter range $\omega_{H}, \omega_{L}$. Setting of the limit values $\omega_{H}, \omega_{L}$ conceal the risk of loss a part of frequency range of ACS free motion. Additional researches have shown, that it is reasonable to return to obtaining optimal parameters for the filters $\omega_{H}^{*}, \omega_{L}^{*}$ in the case of research of the closed-loop ACS with self-tuning.

Comparative research of the filtration efficiency for all cases of the system in fig. 1, in accordance with the plan of computer experiments, is convenient to carry out using table 1.

Table 1

\begin{tabular}{|c|c|c|c|c|c|}
\hline \multicolumn{3}{|c|}{ ACS with self-tuning options } & \multicolumn{3}{|c|}{ Linear band pass filter } \\
\hline \multirow[b]{2}{*}{$\begin{array}{l}\text { «Virtual» } \\
\mathrm{CO}\end{array}$} & \multirow[b]{2}{*}{$\begin{array}{l}\text { Disturbances } \\
\text { models } f_{c}(t)\end{array}$} & \multirow[b]{2}{*}{$\begin{array}{l}\mathrm{CO} \text { model } \\
\text { parameters }\end{array}$} & $(26)$ & $(27)$ & $(28)$ \\
\hline & & & \multicolumn{3}{|c|}{$\begin{array}{l}\text { The values of the statistical correlation coefficient } \hat{r}_{\tilde{y}_{a}} \tilde{y}_{m a} \\
\qquad / \text { difference of dispersions estimations e1 }\end{array}$} \\
\hline \multirow{9}{*}{$(18)$} & \multirow{3}{*}{$(22)$} & $\tau_{m}=0,7 \mathrm{~s}$ & $0,146 /-0,0083$ & $0,245 /-0,0230$ & $0,334 /-0,0135$ \\
\hline & & $\tau_{m}=1 s$ & $0,241 /-0,0082$ & $0,373 /-0,0239$ & $0,487 /-0,0131$ \\
\hline & & $\tau_{m}=1,3 \mathrm{~s}$ & $0,322 /-0,0079$ & $0,474 /-0,0249$ & $0,602 /-0,0128$ \\
\hline & \multirow{3}{*}{ (23) } & $\tau_{m}=0,7 \mathrm{~s}$ & $0,281 /-0,0241$ & $0,352 /-0,1057$ & $0,397 /-0,0892$ \\
\hline & & $\tau_{m}=1 \mathrm{~s}$ & $0,413 /-0,0251$ & $0,504 /-0,1007$ & $0,561 /-0,0924$ \\
\hline & & $\tau_{m}=1,3 \mathrm{~s}$ & $0,511 /-0,0260$ & $0,611 /-0,0986$ & $0,673 /-0,0957$ \\
\hline & \multirow{3}{*}{ (24) } & $\tau_{m}=0,7 \mathrm{~s}$ & $0,395 /-0,0262$ & $0,451 /-0,1812$ & $0,476 /-0,1315$ \\
\hline & & $\tau_{m}=1 \mathrm{~s}$ & $0,546 /-0,0268$ & $0,616 /-0,1680$ & $0,648 /-0,1402$ \\
\hline & & $\tau_{m}=1,3 s$ & $0,639 /-0,0279$ & $0,712 /-0,1608$ & $0,747 /-0,1491$ \\
\hline \multirow{9}{*}{ (19) } & \multirow{3}{*}{$(22)$} & $\tau_{m}=0,7 \mathrm{~s}$ & $0,129 /-0,0082$ & $0,214 /-0,0232$ & $0,293 /-0,0140$ \\
\hline & & $\tau_{m}=1 s$ & $0,224 /-0,0080$ & $0,342 /-0,0241$ & $0,449 /-0,0137$ \\
\hline & & $\tau_{m}=1,3 \mathrm{~s}$ & $0,311 /-0,0078$ & $0,455 /-0,0250$ & $0,581 /-0,0134$ \\
\hline & \multirow{3}{*}{ (23) } & $\tau_{m}=0,7 \mathrm{~s}$ & $0,240 /-0,0241$ & $0,301 /-0,1118$ & $0,343 /-0,0911$ \\
\hline & & $\tau_{m}=1 s$ & $0,373 /-0,0250$ & $0,456 /-0,1084$ & $0,512 /-0,0947$ \\
\hline & & $\tau_{m}=1,3 \mathrm{~s}$ & $0,487 /-0,0259$ & $0,583 /-0,1072$ & $0,648 /-0,0983$ \\
\hline & \multirow{3}{*}{$(24)$} & $\tau_{m}=0,7 \mathrm{~s}$ & $0,324 /-0,0268$ & $0,375 /-0,1932$ & $0,401 /-0,1393$ \\
\hline & & $\tau_{m}=1 s$ & $0,479 /-0,0277$ & $0,546 /-0,1865$ & $0,580 /-0,1466$ \\
\hline & & $\tau_{m}=1,3 s$ & $0,600 /-0,0288$ & $0,676 /-0,1838$ & $0,715 /-0,1540$ \\
\hline \multirow{6}{*}{$(20)$} & \multirow{3}{*}{$(22)$} & $\tau_{m}=0,7 \mathrm{~s}$ & $0,137 /-0,0084$ & $0,226 /-0,0237$ & $0,306 /-0,0144$ \\
\hline & & $\tau_{m}=1 \mathrm{~s}$ & $0,234 /-0,0081$ & $0,358 /-0,0247$ & $0,465 /-0,0143$ \\
\hline & & $\tau_{m}=1,3 s$ & $0,321 /-0,0079$ & $0,471 /-0,0256$ & $0,598 /-0,0141$ \\
\hline & \multirow{3}{*}{ (23) } & $\tau_{m}=0,7 \mathrm{~s}$ & $0,255 /-0,0246$ & $0,317 /-0,1132$ & $0,358 /-0,0957$ \\
\hline & & $\tau_{m}=1 s$ & $0,392 /-0,0256$ & $0,475 /-0,1112$ & $0,529 /-0,1000$ \\
\hline & & $\tau_{m}=1,3 \mathrm{~s}$ & $0,506 /-0,0264$ & $0,604 /-0,1114$ & $0,666 /-0,1043$ \\
\hline
\end{tabular}




\begin{tabular}{|l|c|c|c|c|c|}
\hline \multirow{3}{*}{$(24)$} & $\tau_{m}=0,7 s$ & $0,344 /-0,0270$ & $0,393 /-0,1923$ & $0,417 /-0,1534$ \\
\cline { 3 - 6 } & $\tau_{m}=1 s$ & $0,503 /-0,0281$ & $0,568 /-0,1894$ & $0,599 /-0,1617$ \\
\cline { 3 - 6 } & $\tau_{m}=1,3 s$ & $0,625 /-0,0294$ & $0,699 /-0,1908$ & $0,735 /-0,1697$ \\
\hline
\end{tabular}

It allows to make following conclusions:

1. As a linear filters for self-tuning ACS can be recommended band pass Butterworth filters not less than the fourth order (not less than the second order of the high-pass filter component).

2. AFC of the band pass filter must "embrace" AFC of the closed-loop ACS through the channel of external disturbances, excluding: a) low-frequency areas where spectral density of the control variable changes under the influence of external coordinate disturbances is located; b) high-frequency area, where spectral density of the ACS free motion is getting lower due to inertia specifics of the CO. Instantiation of these recommendations can be done using optimization of the filter cut-off frequencies parameters in the closed-loop ACS with self-tuning.

3. The value of the statistical correlation coefficient during changes of the $\mathrm{CO}$ order changes not significantly, which allows to use the simple first order model with delay as a mathematical model of the real CO.

4. Increase in the dispersions estimations values difference in the outputs of the filters during impact on the system from coordinate disturbances with the most wide spectral density indicates about lowering of the filtration efficiency from the consequences of their impact on the control variables of the $\mathrm{CO}$ and its model. The reason behind this, in this case, is violation of the abovementioned hypothesis about the more low-frequency character of the coordinate disturbances in comparison to the free motion of the ACS frequency character. If this situation takes place in real life, than according high-frequency part of the $\mathrm{CO}$ control variable changes spectrum must be ascribed to measurement noises and must be filtered.

5. The values of the statistical correlation coefficient depend on not precise reflections of the dynamic properties of the $\mathrm{CO}$ in its model, in particular, on not precise time delay setting in the model of $\mathrm{CO}$ in comparison to the time delay in the "virtual" CO. However, arising phase shifts between CO variables and its model variables don't have much influence on dispersions estimations difference, because these estimations are being calculated by using averaging procedure in the time interval, which is greater than the time delay.

Filtration efficiency promotion and, as a consequence, lowering of dispersions estimations difference of the filtered variables can be expected using the decisions, which leads to revelation in these variables of described above negative factors and eliminate their consequences. These decisions needs special design and research.

\section{References}

[1] Rotach V.Y. Avtomatizaciya nastroiki system upravleniya/ V.Y. Rotach, V.F. Kuzish'in, A.S. Kluev - M.: Energoatomizdat, 1984. - 272p.

[2] Khobin V.A. Sistemi garantiruyush'ego upravleniya technologicheskimi agregatami: osnovi teorii, practika primeneniya/V.A. Hobin - Odessa.: TES, 2008. - 306p.

[3] Aizerman M.A. Osnovi avtomaticheskogo regulirovaniya/M.A. Aizerman, V.V. Solodovnikov - M.: Mashgiz, 1954. $1117 \mathrm{p}$.

[4] Patent na poleznuyu model UA 36671 MPK (2006)G05B13/02, released 10.11.2008, bul. 21.

[5] Besekerskiy V.A. Teoriya sistem asvomaticheskogo regulirovaniya/V.A. Besekerskiy, E.P. Popov - M.: Nauka, 1972. $768 \mathrm{p}$.

[6] Livshic N.A. Veroyatnostniy analiz sitem avtomaticheskogo upravleniya/ N.A. Livshic, V.N Pugachev - M.: Sovetskoe radio, 1963. $-896 \mathrm{p}$.

[7] Kulikov E.I. Metodi izmereniya sluchainih processov /E.I. Kulikov - M.: Radio i svyaz', 1986. - 282p.

[8] Levinskyi M.V. Testovie SAR dlya issledovaniya algoritmov ih samonastroiki /M.V. Levinskyi - Odessa.: Naukovi praci ONAHT: vipusk 48/2015. - p. 142-146.

[9] Titze U. Poluprovodnikovaya shemotechnika /U. Titze, K. Shenk -M.: DMK, 2007. - 942p. 